\title{
An iPhone-Assisted Particle Repositioning Maneuver for Benign Paroxysmal Positional Vertigo (BPPV): A Prospective Randomized Study
}

\author{
Brock Organ, MD, BSc, Hao Liu, BSc, and Matthew Bromwich, MD, BSc
}

Purpose: The Epley particle repositioning maneuver (PRM) is an effective treatment for benign paroxysmal positional vertigo (BPPV), the most common cause of peripheral vertigo in primary care settings. The goal of this study was to determine whether the use of an iPhone application (DizzyFIX; Clearwater Clinical Ltd, Ottawa, Ontario, Canada) by medical students had a significant impact on the performance of the PRM.

Methods: We recruited senior medical students who had previously been trained in the management of BPPV and asked them to perform the PRM on a healthy volunteer. One half of the students used a real iPhone application, whereas the others used a sham application. The PRM performance scores of the 2 groups were compared.

Results: iPhone application users scored significantly higher on their PRM performance compared with controls $(P<.0001)$ and performed the PRM significantly more slowly $(P<.0001)$.

Conclusions: Senior medical students performed a more correct PRM when assisted by the iPhone application. This application represents a significant improvement from standard medical school training using written instructions. Family physicians could also use this iPhone application for the quick and effective treatment of BPPV. (J Am Board Fam Med 2015;28:118-120.)

Keywords: Benign Paroxysmal Positional Vertigo, Delivery of Health Care, Graduate Education, Mobile Applications, Otolaryngology

Benign paroxysmal positional vertigo (BPPV) is the most common cause of peripheral vertigo in primary care settings. ${ }^{1}$ The Epley particle repositioning maneuver (PRM) has proven to be a fast, safe, and effective treatment for BPPV., ${ }^{2,3}$

This article was externally peer reviewed.

Submitted 2 November 2012; revised 5 June 2014; accepted 26 September 2014.

From the Faculty of Medicine, University of Ottawa, Ottawa, Ontario, Canada (BO, HL); and the Division of Otolaryngology, Head and Neck Surgery, Children's Hospital of Eastern Ontario, Ottawa, Ontario, Canada (MB).

Funding: none.

Prior presentation: This work was presented as a poster at the American Academy of Otolaryngology-Head and Neck Surgery; September 11-14, 2011; San Francisco, CA.

Conflict of interest: The senior author (MB) now maintains the iPhone app in the iTunes store as a paid app.

Corresponding author: Matthew Bromwich, MD, Division of Otolaryngology, Head and Neck Surgery, Children's Hospital of Eastern Ontario, 451 Smyth Road, Ottawa, ON, Canada K1N 6N5 (E-mail: mbromwich@ cheo.on.ca).
However, studies show that treatment success was only $34 \%$ after a single treatment when administered by family physicians, compared with the $67-89 \%$ success when administered by specialists. $^{2,4}$ The purpose of our study was to determine whether the use of an iPhone application that assists the user in the performance of the PRM had any significant impact on the overall correctness of the performed PRM.

\section{Methods}

The iPhone application DizzyFIX (Clearwater Clinical Ltd, Ottawa, Ontario, Canada) uses the accelerometers and sensors in the iPhone to provide instantaneous feedback as to the orientation of the phone when placed against a patient's head. The application then guides the user through a perfectly correct maneuver (Figure 1). This study was conducted as a prospective, randomized, blinded, placebo-controlled trial. Ethical approval was obtained from the Ottawa Hos- 
Figure 1. Screenshot of the DizzyFIX application. Note the timer, gravity-sensitive head animation, and schematic of the vestibular apparatus with otoconia. Printed with the permission of Clearwater Clinical Ltd.

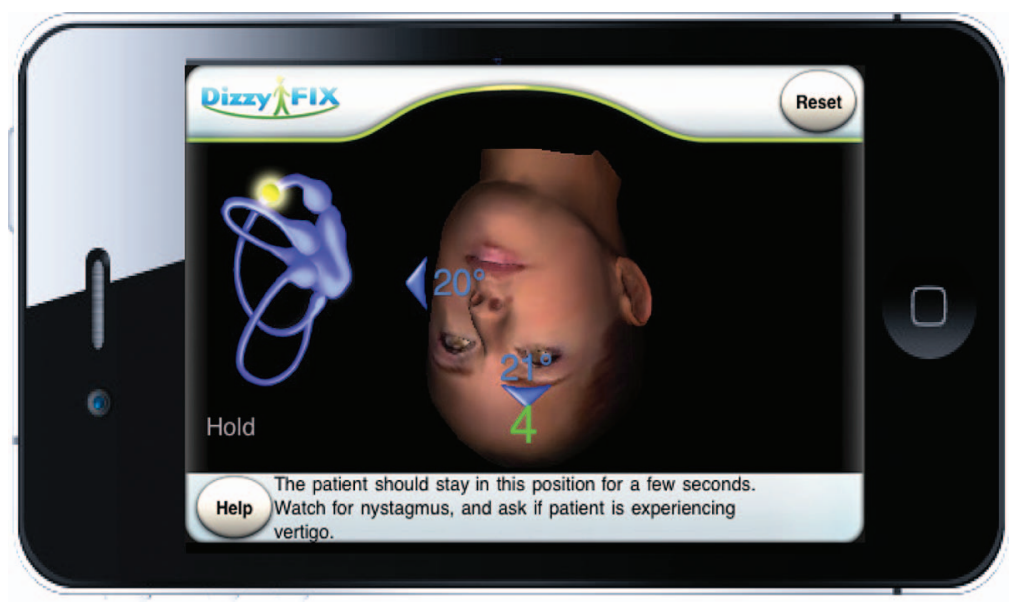

pital Research Ethics Board. The senior author (MB) now maintains the iPhone app on iTunes, although at the time of this research no such conflict of interest existed.

A required sample size of 40 was calculated for nonparametric data. We recruited 41 third-year medical students at the University of Ottawa who had previously received training in the management of BPPV. We randomized the participants into placebo and test groups. Those in the placebo group were given an iPhone with a nonfunctional placebo application and asked to ignore it. This was meant to blind the observer who scored the participants. Those in the test group were given an iPhone with a normally functioning application. Both groups then were asked to perform the maneuver on a healthy volunteer using the application and their previous training. They were scored on the correctness of the PRM and timed in each position by a blinded observer.

\section{Results}

We compared the placebo group's PRM correctness score and the duration of each position against those of the test group. All data were analyzed using SPSS Statistics 17.0 (SPSS Inc., Chicago, IL) The placebo group scored significantly lower on maneuver correctness (mean score, 4.67 of 11 ) than the test group (mean score, 9.65 of 11$)$, with a mean difference of 4.98 points $(P<.0001)$. In addition, the placebo group performed the maneuver faster $(P<.0001)$ than the test group. The majority in the test group kept the volunteer in each position for $>10$ seconds, whereas fewer did so in the unassisted group $(P<.0001)$ (Table 1$)$.

\section{Discussion}

We found that when guided by a PRM iPhone application, third-year medical students performed a much more correct maneuver and stayed in each position longer than those who performed the PRM without assistance from the

Table 1. Participants Who Spent $>10$ Seconds in Each Position of the Particle Repositioning Maneuver, With and Without the iPhone Application

\begin{tabular}{|c|c|c|}
\hline \multirow[b]{2}{*}{ Position } & \multicolumn{2}{|c|}{$\begin{array}{l}\text { Participants Who Spent } \\
>10 \text { Seconds in Each } \\
\text { Position of the PRM }\end{array}$} \\
\hline & $\begin{array}{l}\text { Assisted by } \\
\text { Application } \\
(\mathrm{n}=20)\end{array}$ & $\begin{array}{l}\text { Unassisted } \\
(\mathrm{n}=21)\end{array}$ \\
\hline $\begin{array}{l}\text { 1. Patient sitting position (begins } \\
\text { PRM) }\end{array}$ & N/A & N/A \\
\hline $\begin{array}{l}\text { 2. Ipsilateral head-hanging position } \\
\text { (20-degree extension, 45-degree } \\
\text { ipsilateral rotation) }\end{array}$ & 20 & 8 \\
\hline $\begin{array}{l}\text { 3. Contralateral head-hanging } \\
\text { position (20-degree extension, } \\
\text { 45-degree contralateral rotation) }\end{array}$ & 19 & 5 \\
\hline $\begin{array}{l}\text { 4. Contralateral floor-facing } \\
\text { position (135-degree } \\
\text { contralateral rotation) }\end{array}$ & 18 & 4 \\
\hline $\begin{array}{l}\text { 5. Perpendicular patient-sitting } \\
\text { position (ends PRM) }\end{array}$ & N/A & N/A \\
\hline
\end{tabular}

N/A, not applicable; PRM, particle repositioning maneuver. 
application. Although this study did not explicitly demonstrate that a more correct PRM, as guided by the iPhone application, translates into higher treatment success, it is a reasonable assumption. There are, however, a few other limitations to this study. While the participants' lack of familiarity with the iPhone application and their opportunity to review the PRM in advance may have created bias against the test group, some participants in the unassisted group were unable to complete the maneuver, and thus a short time was recorded, which affected the average. We did not evaluate the usability and user satisfaction of the iPhone application.

\section{Conclusions}

Our results demonstrate the value of this iPhone application as a teaching tool for medical students. Moreover, physicians could use this appli- cation to effectively treat BPPV within their own practice and avoid referring patients with this common condition.

\section{References}

1. Hanley K, O’Dowd T, Considine N. A systematic review of vertigo in primary care. Br J Gen Pract 2001;51:666-71.

2. Froehling DA, Bowen JM, Mohr DN, et al. The canalith repositioning procedure for the treatment of benign paroxysmal positional vertigo: a randomized controlled trial. Mayo Clin Proc 2000;75: 695-700.

3. Radtke A, von Brevern M, Tiel-Wilck K, MainzPerchalla A, Neuhauser H, Lempert T. Self-treatment of benign paroxysmal positional vertigo: Semont maneuver vs Epley procedure. Neurology 2004;63:150-2.

4. Lynn S, Pool A, Rose D, Brey R, Suman V. Randomized trial of the canalith repositioning procedure. Otolaryngol Head Neck Surg 1995;113:712-20. 